\title{
Numerical Taxonomy of the Genus Nocardia
}

\author{
By M. TSUKAMURA \\ The National Sanatorium, Chubu Chest Hospital, \\ Obu, Chita-gun, Aichi-ken 474, Japan
}

(Accepted for publication 3 February 1969)

\section{SUMMARY}

By a numerical classification, strains received as Nocardia asteroides were divided into two clusters; these two groups were named as $N$. asteroides (Eppinger) Blanchard and $N$. farcinica Trevisan, respectively, recognizing the name of $N$. farcinica for the group which included strain ATCC 33I8, received as $N$. asteroides.

The genus Nocardia was divided into 7 groups; (I) N. asteroides (in a new sense); (2) N. brasiliensis; (3) N. caviae; (4) N. farcinica; (5) a group provisionally named $N$. rubra; (6) N. pelletieri; (7) N. madurae.

The species provisionally named $N$. rubra was made up of strains which, when received from other workers, bore 8 species names: $N$. rubra, $N$. erythropolis, N. globerula, N. convoluta, N. minima, N. lutea, N. rubropertincta and $N$. polychromogenes.

Relationships between seven species of Nocardia are shown as a dendrogram, and descriptions of characteristics (here called Hypothetical Mean Organism) of the species are presented.

A method called numerical identification is presented by which identification can be made, without subjective judgement, by comparison of the characters of a test strain with the Hypothetical Mean Organism after permissible limits of S-values for the Hypothetical Mean Organism have been established.

\section{INTRODUCTION}

After the publication of Bergey's Manual of Determinative Bacteriology (1957), the taxonomy of the genus Nocardia was advanced by Gordon \& Mihm (1957, 1959, I $962 a, b$ ) and Gordon ( I $966 a, b$ ), who characterized the species by biochemical tests. In other groups of organisms numerical taxonomy (Sneath, 1957a, $b$, I 964 ; Sneath \& Cowan, 1958; Sokal \& Sneath, 1963) has made a contribution to the elucidation of taxonomic problems, and since no numerical classification has been made on the genus Nocardia (see Appendix), it was decided to apply this method to the genus.

\section{METHODS}

One hundred and twelve strains received as nocardiae were used for the study. Thirty-four strains were received in 1967 from Dr Ruth E. Gordon, Institute of Microbiology, Rutgers University, New Brunswick, New Jersey, U.S.A., under the following species names: $N$. asteroides (IO strains); $N$. brasiliensis (7 strains); $N$. caviae ( 7 strains); N. pelletieri (5 strains); and $N$. madurae (5 strains). For these strains we retained the strain names. The other 78 strains were received in 1965 and 1967 from Dr I. Uesaka, Tuberculosis Research Institute, Kyoto University, Kyoto, Japan;

Vol. 56, No. 2, was issued 17 June 1969 
among these, 66 had been supplied to Dr Uesaka by Dr N. M. McClung, University of South Florida, Tampa, Florida, U.S.A., and these 66 strains were designated by the letter M. Eight strains had been supplied to Dr Uesaka by Dr McClung through Dr Arai, Chiba University, Chiba, Japan. These also were designated with the letter $\mathrm{M}$. Four strains had been supplied to Dr Uesaka by Dr Gordon through Dr Arai; these were designated by the letter C. During investigations in our laboratory the strains were given a laboratory number so that their identities were concealed.

All strains were subcultured on glucose agar and kept at $-20^{\circ}$. The composition of the glucose agar was as follows: glucose, Io g.; sodium glutamate, $4.0 \mathrm{~g}$.; $\mathrm{KH}_{2} \mathrm{PO}_{4}$, $0.5 \mathrm{~g}$.; $\mathrm{MgSO}_{4} .7 \mathrm{H}_{2} \mathrm{O}$, $0.5 \mathrm{~g}$.; agar, $20.0 \mathrm{~g}$.; distilled water, $\mathrm{I}, 000 \mathrm{ml}$; adjusted to $\mathrm{pH} 7.0$ by addition of $10 \%(\mathrm{w} / \mathrm{v}) \mathrm{KOH}$.

One hundred characters were tested (see below); the methods employed were described previously (Tsukamura, 1967a); incubation was at $28^{\circ}$, unless specially noted.

Among the I00 characters 97 were set up originally for differentiating between the members of the genus Mycobacterium. Only 3 other characters were added; these were: (I) partial acid-fastness; (2) permanent mycelium; and (3) temporary mycelium.

Morphological characters were observed on smears from organisms growing on Ogawa egg medium or glucose agar, and on slide cultures of glucose agar after I, 2, 3, and 7 days of incubation, by the Ziehl-Neelsen method. The term 'permanent' mycelium was used for well-developed mycelium showing fragmentation to rods or coccoids, mycelia being observed throughout the incubation period; 'temporary' mycelium for mycelia visible at an early stage of growth which soon became rods and coccoids. The term 'complete' acid-fastness was applied to organisms as acidfast as growing mycobacteria.

Amidase tests were done according to the method of Bönicke (1962), the time of incubation being modified to $\mathrm{I} 6 \mathrm{hr}$ at $37^{\circ}$. To avoid contamination of the organisms with components of medium in the amidase tests, only glucose agar-grown cultures were used. Egg media seemed to be unsuitable for nocardiae, producing growth adherent to egg media.

All tests were done three times and the results obtained in at least two replications were used for preparation of charts of characters. The results were expressed by symbols $(+,-)$, and the S-value was calculated as follows:

$$
\text { S-value }(\%)=\left(n_{s} / n_{s}+n_{d}\right) \times 100 \% \text {, }
$$

where $n_{s}$ is the number of characters in which two strains showed similar code symbols $(++$ or --$)$ and $n_{d}$ is the number of characters in which two strains showed different symbols $(+-)$. The method used for calculation of S-values was described previously (Tsukamura, I966). Clustering was made by single linkage (Sokal \& Sneath, 1963).

Among the characters used 25 were ineffective for differentiation between the members of the genus Nocardia, giving either all positive or all negative results for the whole set of organisms. Nevertheless, these were retained in the test series. On the other hand, several characters reported as useful for differentiation between the members of the genus Nocardia (Gordon \& Mihm, I957, 1962a) were not included in this study because the same test series was applied to both mycobacteria and nocardias.

Hypothetical Mean Organism (HMO) is the term used to express a mean charac- 
terization of a species, which is a slight modification of the 'Calculated Median Organism' of Liston, Wiebe \& Colwell (1963).

For preparation of HMOs, the mean number of positive characters (mean of the characters showing a positive reaction in a strain) is calculated for every species or group. The number of positive characters in an HMO is equal to the mean number of positive characters per strain. The characters to be used for preparation of an HMO are chosen, first taking a character appearing at the highest frequency and then taking a character appearing at the next (lower) frequency, and the choice of such positive characters continues until the number of characters obtained equals the mean number of positive characters.

The number of characters used for the preparation of HMOs is not always equal to the mean number. If, after selection of one less than the mean number of characters, three characters showing the same frequency are found, these three are added, obtaining two characters more than the mean number. The HMOs thus prepared are similar to the Calculated Median Organisms of Liston et al. (I963). The average S-value between the HMO and the Calculated Median Organism in various mycobacterial species was $98.5 \%$ (Tsukamura \& Mizuno, I968). Details of the method of preparation of the HMO and a few advantages in the use of the HMO (for example, it is possible to calculate the degree of deviation in the characters of an HMO) were described previously (Tsukamura \& Mizuno, I968).

\section{RESULTS}

\section{Numerical classification of Nocardia (strains provided by Dr Gordon)}

Numerical classification of 34 strains of Nocardia received from $\mathrm{Dr}$ Gordon as reference strains showed clearly 6 clusters: $(\mathrm{I}, 2) N$. asteroides (two clusters); (3) $N$. brasiliensis; (4) N. caviae; (5) N. pelletieri; (6) N. madurae (Fig. I). The clusters of $N$. asteroides were labelled group A (from ATCC 9970 to R-553) and group B (from R-764 to ATCC 33I8), respectively. Strain R-I35I received as $N$. caviae was not in the cluster of $N$. caviae and, therefore, was omitted from the group of $N$. caviae.

A dendrogram showing relationships between the species was prepared from the S-value table shown in Fig. I and is shown in Fig. 2.

Since a type strain of Nocardia asteroides seems to be non-existent, it was desired to consider which group contained, as nearly as possible, the characters attributed to $N$. asteroides. All strains showed the characters described by Gordon \& Mihm (I957, I959, 1962 a), but three characters used by them were variable. Not all of the 7 strains of group A formed acid from rhamnose, or grew at $45^{\circ}$, and 5 of 7 showed reduction of nitrate to nitrite. On the other hand, all 3 strains of group B formed acid from rhamnose, grew at $45^{\circ}$, and failed to reduce nitrate. Gordon \& Mihm (I957, 1959, 1962a) described these characters as follows: (a) $32 \%$ of the strains tested formed acid from rhamnose; $(b) 42 \%$ of the strains grew at $45^{\circ}$; and $(c) 88 \%$ of the strains reduced nitrate to nitrite (Gordon \& Mihm, 1962a). These characters resemble our group A rather than group B; accordingly, group A was named $N$. asteroides, but group B required another name. Group B included strain ATCC 3318, named $N$. farcinica Trevisan (Gordon \& Mihm, I957, 1962a), which name seemed to be suitable for group B.

Differentiation between groups A and B could be made not only by these characters 


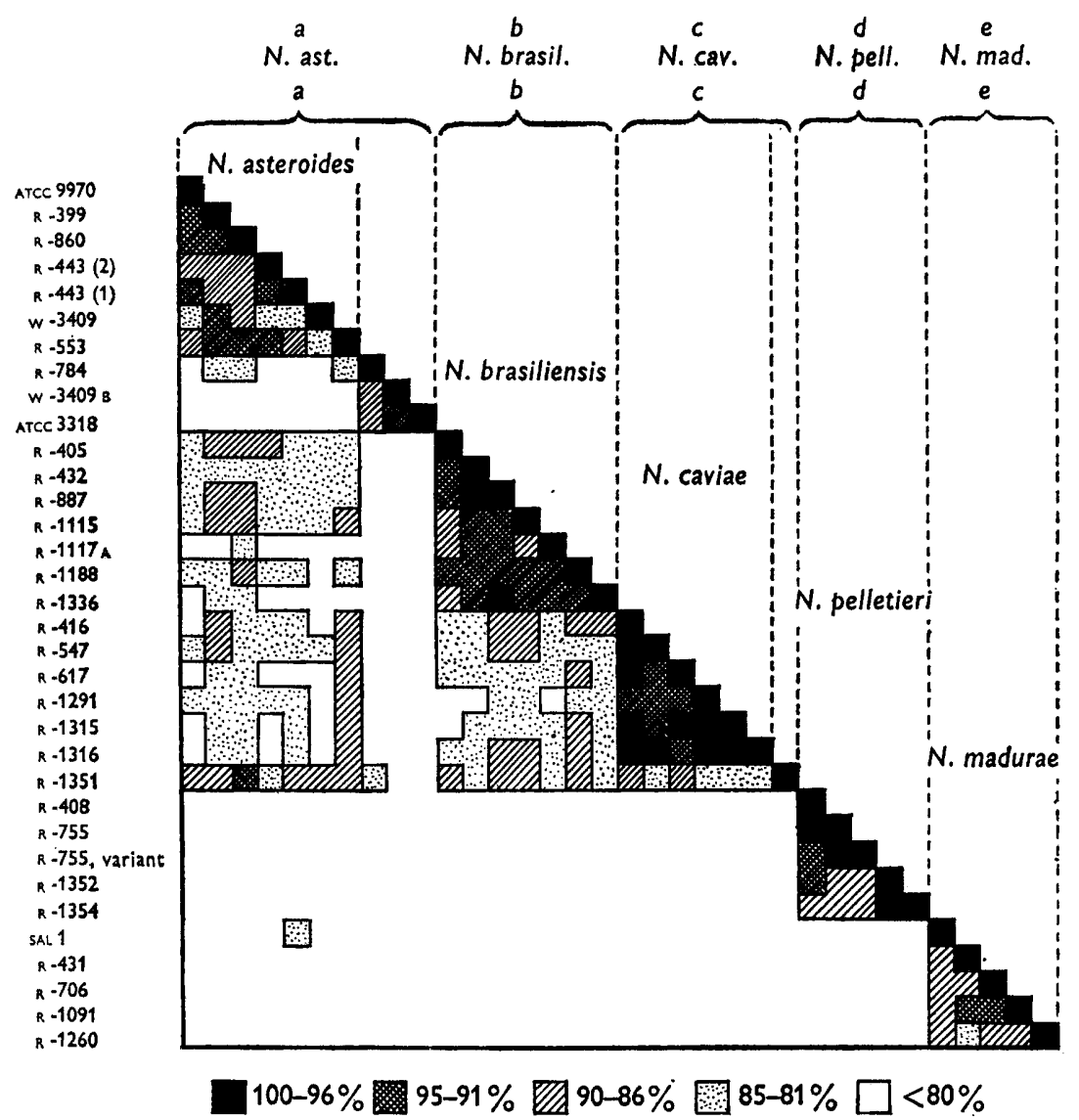

Fig. I. Diagrammatic representation of the S-value table prepared by shading the square of a S-value between the strains.

The species names above are the names received.

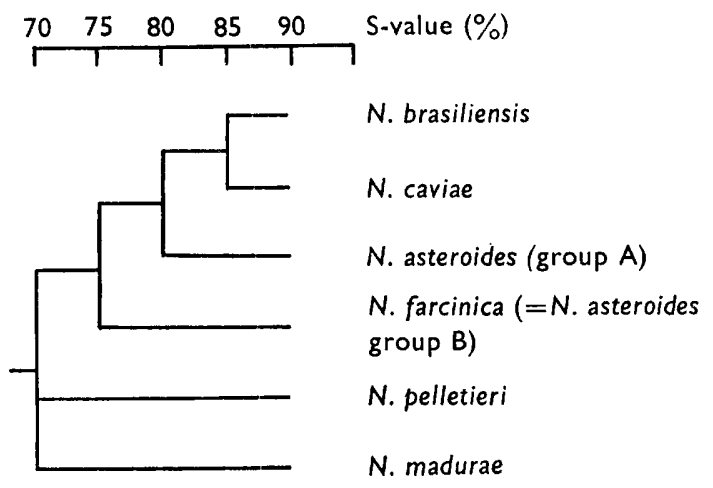

Fig. 2. Dendrogram prepared from the S-value table shown in Fig. I. 
but more distinctly by many other characters (see below). It is now intended to demonstrate differences between these two species in tests of more strains and to make acceptable the proposal for the division of the species $N$. asteroides into two separate species.

Numerical classification of Nocardia (strains provided by Dr Uesaka and Dr McClung)

Results of the numerical classification of 78 strains received from Dr Uesaka, which were mainly from the collection of Dr McClung, are shown in Fig. 3. Two distinct clusters were present, but half of the strains remained unclustered. One of the clusters consisted of 34 strains (from $\mathrm{C}-407$ to $\mathrm{M}-\mathrm{I} 75$ ); the other of 7 strains (from $\mathrm{M}-\mathrm{I}$ to

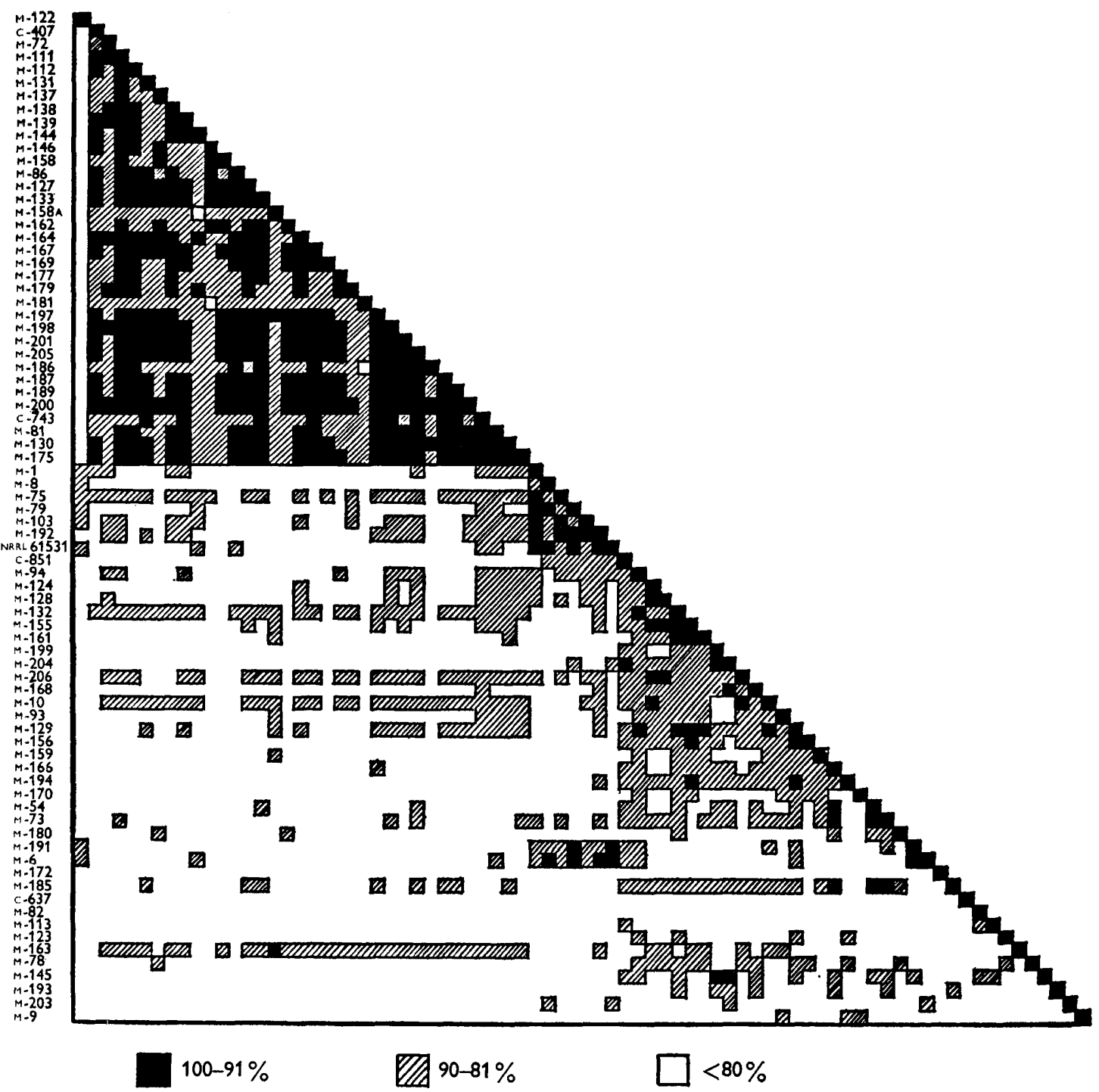

Fig. 3. Diagrammatic representation of the S-value table prepared by shading the square of a S-value between the strains. 
Table I. 'Hypothetical Mean Organisms' (HMOs) of the species of the genus Nocardia (comparison with Table 2)

I. Gram reaction

2. Acid-fastness, complete

3. Acid-fastness, partial

4. Mycelium, permanent

5. Mycelium, temporary

6. Rod shape (with or without coccoids)

7. Cord formation (compact grouping)

8. Colonial morphology, rough

9. Colonial pigmentation

10. Photochromogenicity

II. Growth rate, rapid

12. Catalase

13. Nitrate reduction

14. Three-day arylsulphatase

15. Two-week arylsulphatase

16. Salicylate degradation

17. PAS degradation

18. Growth on $0.2 \%$ PAS medium

19. Growth on $\mathrm{NH}_{2} \mathrm{OH}$ medium (62.5 $\mu \mathrm{g} . / \mathrm{ml}$.)

20. Growth on $\mathrm{NH}_{2} \mathrm{OH}$ medium ( $25 \mu \mathrm{g} . / \mathrm{ml}$.)

21. Growth on $\mathrm{NH}_{2} \mathrm{OH}$ medium $(250 \mu \mathrm{g} . / \mathrm{ml}$.)

22. Growth on $\mathrm{NH}_{2} \mathrm{OH}$ medium $(500 \mu \mathrm{g} . / \mathrm{ml}$.

23. Tolerance to $0.1 \%$ picric acid

24 . Tolerance to $0.2 \%$ picric acid

25. Growth at $28^{\circ}$

26. Growth at $37^{\circ}$

27. Growth at $45^{\circ}$

28. Growth at $52^{\circ}$

29. Acetamidase

30. Benzamidase

31. Urease

32. Isonicotinamidase

33. Nicotinamidase

34. Pyrazinamidase

35. Salicylamidase

36. Allantoinase

37. Succinamidase

38. Malonamidase

39. Acetate (as C source)

40. Citrate (as $\mathrm{C}$ source)

41. Succinate (as C source)

42. Malate (as $\mathrm{C}$ source)

43. Pyruvate (as $\mathrm{C}$ source)

44. Benzoate (as $\mathrm{C}$ source)

45. Malonate (as $C$ source)

46. Fumarate (as $\mathrm{C}$ source)

47. Acid from glucose

48. Acid from mannose

49. Acid from galactose

50. Acid from arabinose

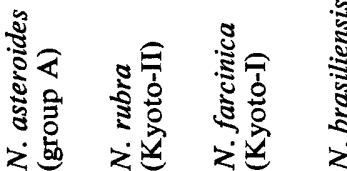


Table I (cont.)

5I. Acid from xylose

52. Acid from rhamnose

53. Acid from trehalose

54. Acid from raffinose

55. Acid from inositol

56. Acid from mannitol

57. Acid from sorbitol

58. Glycerol (as C source)

59. Glucose (as C source)

60. Mannose (as $\mathrm{C}$ source)

61. Galactose (as $C$ source)

62. Arabinose (as $\mathrm{C}$ source)

63. Xylose (as C source)

64. Rhamnose (as C source)

65. Trehalose (as $\mathrm{C}$ source)

66. Raffinose (as $\mathrm{C}$ source)

67. Inositol (as $\mathrm{C}$ source)

68. Mannitol (as C source)

69. Sorbitol (as C source)

70. Fructose (as $\mathrm{C}$ source)

71. Sucrose (as C source)

72. Ethanol (as C source)

73. Propanol (as $\mathrm{C}$ source)

74. Propylene glycol (as $\mathrm{C}$ source)

75. I,3-Butylene glycol (as C source)

76. 1,4-Butylene glycol (as C source)

77. 2,3-Butylene glycol (as C source)

78. L-Glutamate (as $\mathrm{N}$ and $\mathrm{C}$ sources)

79. L-Serine (as $\mathrm{N}$ and $\mathrm{C}$ sources)

8o. Glucosamine (as $\mathrm{N}$ and $\mathrm{C}$ sources)

81. Acetamide (as $N$ and $C$ sources)

82. Benzamide (as $\mathrm{N}$ and $\mathrm{C}$ sources)

83. Monoethanolamine (as $\mathrm{N}$ and $\mathrm{C}$ sources)

84. Trimethylene diamine (as $\mathrm{N}$ and $\mathrm{C}$ sources)

85. L-Glutamate (as $\mathrm{N}$ source)

86. L-Serine (as $N$ source)

87. L-Methionine (as N source)

88. Acetamide (as $N$ source)

89. Benzamide (as $\mathrm{N}$ source)

90. Urea (as N source)

91. Pyrazinamide (as $\mathrm{N}$ source)

92. Isonicotinamide (as $\mathrm{N}$ source)

93. Nicotinamide (as $\mathrm{N}$ source)

94. Succinamide (as $\mathrm{N}$ source)

95. Nitrate (as $N$ source)

96. Nitrite (as $\mathrm{N}$ source)

97. Nicotinic acid
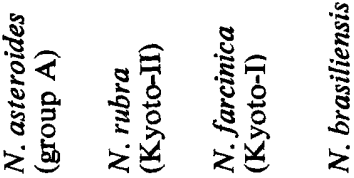

99. Growth on salicylate medium (0.05\%)

I00. Growth on salicylate medium $(0.1 \%)$ 
NRRL 6153I). As shown later, strains of the large group were identical with group B, that is, $N$. farcinica, and another group was shown to be a species distinguishable from the other species. In the following, the course of classification and identification will be described in detail.

Table 2. Properties of 7 groups of Nocardia (mean no. of positive characters, intragroup-S-value for each group, and mean $S$-value to the HMO in each group)

\begin{tabular}{|c|c|c|c|c|}
\hline Group & $\begin{array}{l}\text { No. of } \\
\text { strains }\end{array}$ & $\begin{array}{c}\text { Mean no. of } \\
\text { positive characters }\end{array}$ & $\begin{array}{c}\text { Intragroup-S-value } \\
(\%)\end{array}$ & $\begin{array}{c}\text { Mean S-value to } \\
\text { the HMO }(\%)\end{array}$ \\
\hline $\begin{array}{l}N . \text { asteroides (group A } \\
\text { of } N . \text { asteroides) }\end{array}$ & 7 & $\begin{array}{c}44 \cdot 3 \pm 4 \cdot 03 \\
(n=7)\end{array}$ & $\begin{array}{c}89 \cdot 1 \pm 3 \cdot 38 \\
(n=2 \mathrm{I})\end{array}$ & $\begin{array}{c}93 \cdot 6 \pm 2 \cdot 58 \\
(n=7)\end{array}$ \\
\hline $\begin{array}{l}\text { N. rubra (group } \\
\text { Kyoto-II) }\end{array}$ & 7 & $\begin{array}{c}56 \cdot 6 \pm 3 \cdot 05 \\
(n=7)\end{array}$ & $\begin{array}{l}9 \mathrm{I} \cdot 9 \pm 2 \cdot 74 \\
(n=2 \mathrm{I})\end{array}$ & $\begin{array}{l}95 \cdot 3 \pm \mathrm{I} \cdot 98 \\
(n=7)\end{array}$ \\
\hline $\begin{array}{l}\text { N. farcinica (group } \\
\text { Kyoto-I) }\end{array}$ & 30 & $\begin{array}{c}52 \cdot 7 \pm 4 \cdot 26 \\
(n=30)\end{array}$ & $\begin{array}{c}9 \mathrm{I} \cdot 0 \pm 3 \cdot 87 \\
(n=435)\end{array}$ & $\begin{array}{c}94 \cdot 6 \pm 3 \cdot 17 \\
(n=30)\end{array}$ \\
\hline N. brasiliensis & 7 & $\begin{array}{c}48 \cdot 3 \pm 3 \cdot 15 \\
(n=7)\end{array}$ & $\begin{array}{c}92 \cdot 8 \pm 2 \cdot 25 \\
(n=21)\end{array}$ & $\begin{array}{c}95 \cdot 9 \pm 2 \cdot 55 \\
(n=7)\end{array}$ \\
\hline$N$. caviae & 6 & $\begin{array}{c}52 \cdot 5 \pm 2 \cdot 43 \\
(n=6)\end{array}$ & $\begin{array}{c}96 \cdot 0 \pm 1 \cdot 46 \\
(n=15)\end{array}$ & $\begin{array}{c}97 \cdot 5 \pm \mathrm{I} \cdot 38 \\
(n=6)\end{array}$ \\
\hline$N$. pelletieri & 5 & $\begin{array}{l}20 \cdot 8 \pm \mathrm{I} \cdot 48^{*} \\
(n=5)\end{array}$ & $\begin{array}{c}92 \cdot 2 \pm 3 \cdot 84 \\
(n=10)\end{array}$ & $\begin{array}{c}96 \cdot 6 \pm \mathrm{I} \cdot 34 \\
(n=5)\end{array}$ \\
\hline N. madurae & 5 & $\begin{array}{c}46 \cdot 8 \pm 3 \cdot 1 \mathrm{I}^{*} \\
(n=5)\end{array}$ & $\begin{array}{c}89 \cdot 5 \pm 2 \cdot 22 \\
(n=10)\end{array}$ & $\begin{array}{c}92 \cdot 4 \pm 2 \cdot 07 \\
(n=5)\end{array}$ \\
\hline
\end{tabular}

* Data on egg media were excluded. Accordingly, a total of 91 characters was tested. In other groups, a total of 100 characters was tested.

Table 3. S-value table (\%) of the 'Hypothetical Mean Organisms' (HMOs)

of 7 groups of Nocardia

$\begin{array}{llllll}(1) & (2) & \text { (3) } & \text { (4) } & \text { (5) } & \text { (6) }\end{array}$

(1) N. asteroides (group A)

(2) N. brasiliensis

(3) N. caviae

(4) N. rubra (Kyoto-II)

(5) N. farcinica (Kyoto-I)

(6) N. madurae

(7) N. pelletieri
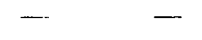

-
-
-
-
79
78
64

-
-
-
-
-
70
69

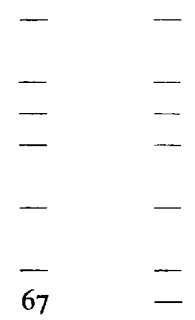

Intragroup-mean S-values to the HMO are as follows: (I) $93.6 \pm 2.58 \%(n=7) ;(2) 95.9 \pm 2.55 \%$ $(n=7)$; (3) $97 \cdot 5 \pm \mathrm{I} \cdot 38 \%(n=6)$; (4) $95 \cdot 3 \pm \mathrm{I} \cdot 98 \%(n=7) ;(5) 94 \cdot 6 \pm 3 \cdot 17 \%(n=30) ;(6) 92 \cdot 4 \pm$ $2.07 \%(n=5) ;(7) 96 \cdot 6 \pm \mathrm{I} \cdot 34 \%(n=5)$. (Refer to the right column in Table 2.)

Figure 3 was prepared by a rearrangement of the S-value table of 78 strains. When a diagrammatic representation of the S-value table of the strains was first prepared, two clusters were recognized, one large cluster consisting of 30 strains (from C-407 to M-200) and another small cluster consisting of 7 strains (from M-I to NRRL 6I53I). These clusters were named provisionally Kyoto-I and Kyoto-II, respectively. In order to compare these two groups with the 6 groups obtained by a numerical classification of Dr Gordon's strains, HMOs were prepared for each of the following groups (Table I): (I) Kyoto-I; (2) Kyoto-II ; (3) Nocardia asteroides (group A); (4) N. brasili- 
ensis; (5) N. caviae; (6) N. pelletieri; (7) N. madurae (Table I). The strains used for preparation of the HMOs were as follows: (I) Kyoto-I (30 strains, C-407 (received as $N$. madurae) to M-200 (received as $N$. brasiliensis) in Fig. 3); (2) Kyoto-II (7 strains,

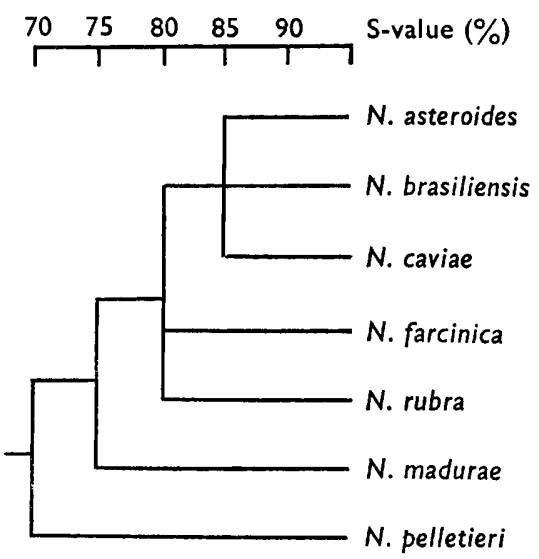

Fig. 4. Dendogram prepared from the S-value table of HMOs of the species of Nocardia.

Table 4. Comparison between Nocardia asteroides (group A) and $N$. brasiliensis

$\begin{array}{ccc} & \begin{array}{c}N . \text { asteroides } \\ \text { (group A) } \\ (\%)\end{array} & \begin{array}{c}N . \text { brasiliensis } \\ (\%)\end{array} \\ \text { Mean S-value to own HMO } & 93 \cdot 6 \pm 2 \cdot 58 & 95 \cdot 7 \pm 2 \cdot 55 \\ & (n=7) & (n=7) \\ \text { Mean S-value to other HMO } & 83 \cdot 7 \pm 2 \cdot 0 \mathrm{I} & 86 \cdot 4 \pm 2 \cdot 5 \mathrm{I} \\ & (n=7) & (n=7) \\ & & \end{array}$

Table 5. Intra- and inter-groups mean $S$-values, Nocardia asteroides and N. brasiliensis
(I)
$(\%)$
(2)
$(\%)$
(1) N. asteroides (group A) (7 strains)
$89 \cdot 1 \pm 3 \cdot 38$
$(n=2 \mathrm{I})$
(2) N. brasiliensis (7 strains)
$82 \cdot 9 \pm 3 \cdot 37$
$(n=49)$
$92 \cdot 8 \pm 2 \cdot 25$
$(n=2 \mathrm{I})$

Intra-group mean $\mathrm{S}$-values are significantly larger than inter-groups mean S-value ( $P$ less than $5 \%$ ).

M-I (received as $N$. rubra) to NRRL 6I53I (received as $N$. polychromogenes) in Fig. 3); (3) N. asteroides ( 7 strains, ATCC 9970 to R-553 in Fig. I); (4) N. brasiliensis ( 7 strains, R-405 to R-I336 in Fig. I); (5) N. caviae (6 strains, R-4I6 to R-I3I6 in Fig. I); (6) $N$. pelletieri (5 strains in Fig. 1); (7) N. madurae (5 strains in Fig. I). Group B, N. farcinica, was omitted from the above groups, as it consisted of only 3 strains. 
Table 6. Intra- and inter-groups mean $S$-values, Nocardia brasiliensis and $N$. caviae
(I)
$(\%)$
(2)
$(\%)$
(1) $N$. brasiliensis (7 strains)
$92 \cdot 8 \pm 2 \cdot 25$
$(n=2 \mathrm{I})$
(2) N. caviae (6 strains)
$84 \cdot 6 \pm 2 \cdot 62$
$(n=42)$
$96 \cdot 0 \pm \mathrm{I} \cdot 46$
$(n=15)$

Intra-group S-values are significantly larger than inter-groups mean S-value ( $P$ less than $5 \%)$.

Table 7. Intra-and inter-groups mean S-values, Kyoto-I and Kyoto-II groups
(I)
$(\%)$
$(2)$
$9 \mathrm{I} \cdot 0 \pm 3 \cdot 87$
(I) Kyoto-I (30 strains)
$(n=435)$
(2) Kyoto-II (7 strains)
$77 \cdot 9 \pm 3 \cdot 87$
$(n=210)$
$9 \mathrm{I} \cdot 9 \pm 2 \cdot 74$
$(n=2 \mathrm{I})$
$(\%)$

Intra-group mean S-values are significantly larger than inter-groups mean S-value $(P$ less than $5 \%)$.

Table 8. Identification of strains ATCC 33I8, W-3409B and R-784

(group $B$ of Nocardia asteroides)

Hypothetical Mean

Organism (HMO)

N. farcinica (Kyoto-I)

N. rubra (Kyoto-In)

$N$. asteroides (group A)

N. brasiliensis

$N$. caviae

$N$. pelletieri

N. madurae

\begin{tabular}{cc}
\multicolumn{2}{c}{ S-value to HMO } \\
ATCC 33 I 8 & W-3409 B \\
93 & 93 \\
78 & 75 \\
77 & 74 \\
74 & 68 \\
71 & 70 \\
70 & 76 \\
73 & 67
\end{tabular}

* The permissible low limit of the S-value to the HMO for members of a species is at (mean) -2 $\times($ standard deviation $) \%$.

Table 9. Strains identified, provisionally, as Nocardia rubra

(Kruse) Chalmers \& Christopherson

Strain*

N. rubra $\mathrm{M}-\mathrm{I}$

$N$. erythropolis $\mathrm{M}-8$

N. globerula $\mathrm{M}-75$

N. convoluta $\mathrm{M}-79$

$N$. minima $\mathrm{M}-\mathrm{IO} 3$

$N$. lutea $\mathrm{M}-192$

N. polychromogenes NRRL 6I53I

$N$. polychromogenes M-6

N. rubropertincta $\mathrm{M}-\mathrm{I} 9 \mathrm{I} \dagger$
History

N. M. McClung; CBS, Holland (= R-562)

N. M. McClung; Waksman (3407)

N. M. McClung; NRRL no. BI 306 (= ATCC $9356=$ R-4I7)

N. M. McClung; ATCC 4275

N. M. McClung; ATCC 8674

N. M. McClung; D. Schneidau (365); R. E. Gordon (R-68o)

E. N. Azarowiez (147); Inst. Pasteur, Paris

N. M. McClung; NRRL 6I53I

N. M. McClung; N. polychromogenes (Vallée) Jensen; CBS; (= R-565)

N. M. McClung; J. D. Schneidau

* Species name is the name when received.

$\dagger$ Suggested to be a member of this species (see Table 10). 
Table Io. Identification by comparison with Hypothetical Mean Organisms $(H M O s)$ of strains not forming a cluster in preliminary sorting

S-value $(\%)$ to the HMO of

Species name and strain name as received

Permissible low limit $(\%) \dagger$

N. coeliaca $\mathrm{M}-\mathrm{I} 22$

$N$. madurae C-743

$N$. blackwellii $\mathrm{M}-8 \mathrm{I}$

$N$. asteroides $\mathrm{M}-\mathrm{I} 30$

$N$. asteroides $\mathrm{M}-\mathrm{I} 63$

N. corrallina $\mathrm{M}-78$

$N$. brasiliensis $\mathrm{C}-85 \mathrm{I}$

$N$. asteroides $\mathrm{M}-94$

$N$. asteroides $\mathrm{M}-\mathrm{I} 24$

$N$. asteroides $\mathrm{M}-\mathrm{I} 28$

$N$. asteroides $\mathrm{M}-\mathrm{I} 32$

$N$. brasiliensis M-145

$N$. asteroides $\mathrm{M}-\mathrm{I} 55$

$N$. asteroides $\mathrm{M}-\mathrm{I} 6 \mathrm{I}$

$N$. transvalensis $\mathrm{M}-\mathrm{I} 93$

$N$. brasiliensis M-199

$N$. brasiliensis $\mathrm{M}-204$

$N$. asteroides $\mathrm{M}-206$

N. brasiliensis $\mathrm{M}-\mathrm{I} 68$

Nocardia sp. M- 54

$N$. asteroides $\mathrm{M}-73$

$N$. asteroides $\mathrm{M}-\mathrm{I} 80$

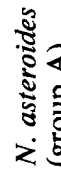

88.4

69

84

83

83

80

86

85

9 I

92

90

93

84

$9 \mathrm{I}$

90

79

83

86

91

87

80

83

75

$N$. tenuis convoluta $\mathrm{M}-203 \quad 78$

$N$. restrictus $\mathrm{M}-9$

$N$. asteroides $\mathrm{M}-\mathrm{IO}$

$N$. asteroides $\mathrm{M}-93$

N. asteroides $\mathrm{M}-\mathrm{I} 29$

$N$. asteroides $\mathrm{M}-\mathrm{I} 56$

$N$. bostroemi M-I 59

$N$. asteroides $\mathrm{M}-\mathrm{I} 66$

$N$. intracellularis $\mathrm{M}-\mathrm{I} 70$

$N$. pretoriana $\mathrm{M}-\mathrm{I} 94$

N. rubropertincta $\mathrm{M}-\mathrm{I} 9 \mathrm{I}$

N. polychromogenes $\mathrm{M} \cdot 6$

N. madurae $\mathrm{M}-\mathrm{I} 72$

$N$. asteroides $\mathrm{M}-175$

$N$. asteroides $\mathrm{M}-\mathrm{I} 85$

$N$. madurae C-637

N. leishmanii $\mathrm{M}-82$

$N$. alba (Schoen) M-I I 3

$N$. asteroides $\mathrm{M}-\mathrm{I} 23$

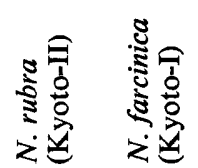

$91 \cdot 3 \quad 88 \cdot 3$

84

83

84

80

77

75

82

82

81

83

80

76

80

75

77

78

79

82

78

77

80

70

80

$6 I$

79

79

79
80

75

71

71
76

76
67

67
77

77
89

89
93
72

80

80
75

75
75

72

72

64

92

95

97

$$
84
$$

74

73

$8 \mathrm{I}$

80

80

85

85
73
79

78

73

75

76

83

83
75
80

$8 \mathrm{I}$

81
75
75

75

64

84

78

81

76

78

79

74

74
74

74
76

73

73
97

80

68

$6 I$

67

68
之ं

90.8

3

$\begin{array}{lllll}71 & 75 & 71 & 50 & 72\end{array}$

$\begin{array}{lll}75 & 71 & 50 \\ 80 & 76 & 68 \\ 77 & 77 & 65 \\ 77 & 77 & 69\end{array}$

$84-77$

$\begin{array}{ll}84 & 92 \\ 65 & 63\end{array}$

$78 \quad 67$

79

71

73
67

52

58

60

85

F

F

F

F

A

B

A

A

A

A

B

A

A

B

B

A

B

C

$\mathrm{C} \ddagger$

.

A

A

A

A

$\mathrm{C} \ddagger$

A

R

R

F

C

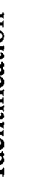

* $\mathrm{A}=N$. asteroides (group $\mathrm{A}) ; \mathrm{R}=N$. rubra (Kyoto-II); $\mathrm{F}=N$. farcinica (Kyoto-I); $\mathrm{B}=$ $N$. brasiliensis $; \mathrm{C}=N$. caviae $; \mathrm{Pe}=N$. pelletieri $; \mathrm{M}=N$. madurae.

$\dagger$ As to the permissible limit of $\mathbf{S}$-value, see Table 5.

$\ddagger$ If an S-value was outside the permissible limit of 7 species but remained within a $5 \%$ range from the permissible limit of a species, this species name was indicated with the double dagger. If an $S$-value was within the permissible low limit of a species, this species name was indicated as the species identified. Spaces remaining blank show that no species was identified. 
Mean number of positive features for each group or species, intragroup-S-value for each group, and mean S-value to the HMO for the members of each group are shown in Table 2.

An S-value table of the HMOs is shown in Table 3, and a dendrogram prepared from it is shown in Fig. 4. Comparing the mean S-values to the HMO, which are shown in Table 2, and the S-values between the HMOs, which are shown in Table 3, it is conceivable that there are statistically significant differences between an intragroup-S-value to the HMO, which is shown on the right column in Table 2, and an intergroup-S-value (Table 3), and these 7 groups were considered to be distinct species.

\section{Table II. Strains identified as Nocardia asteroides (Eppinger) Blanchard (Blanchard, I896)}

\section{Strain*}

N. asteroides ATCC 9970

N. asteroides $\mathrm{R}-399$

$N$. asteroides $\mathrm{R}-860$

$N$. asteroides $\mathrm{R}-443$ (I)

$N$. asteroides $\mathrm{R}-443$ (2)

$N$. asteroides $\mathrm{W}-3409$

$N$. asteroides $\mathrm{R}-553$

N. caviae $\mathrm{R}-135 \mathrm{I}$

$N$. asteroides $\mathrm{M}-94$

$N$. asteroides $\mathrm{M}-\mathrm{I} 24$

$N$. asteroides $\mathrm{M}-\mathrm{I} 28$

$N$. asteroides $\mathrm{M}-\mathrm{I} 32$

$N$. asteroides M-I 55

$N$. asteroides $\mathrm{M}-\mathrm{I} 6 \mathrm{I}$

N. asteroides $\mathrm{M-206}$

N. asteroides $\mathrm{M}-\mathrm{IO}$

$N$. asteroides $\mathrm{M}-93$

$N$. asteroides $\mathrm{M}-\mathrm{I} 29$

N. asteroides M-I 56

$N$. pretoriana $\mathrm{M}-\mathrm{I} 94$

$N$. corallina $\mathrm{M}-78 \dagger$
History

R. E. Gordon; C. W. Emmons (NIH) (9956); C. A. Perry

R. E. Gordon; C. W. Emmons (NIH) (9966)

R. E. Gordon; W. D. Jones, Jun.

R. E. Gordon; I. B. Christison; N. F. Conant (2338)

R. E. Gordon; I. B. Christison; N. F. Conant (2338)

R. E. Gordon; N. polychromogenes; S. A. Waksman; H. L. Jensen

R. E. Gordon

R. E. Gordon

N. M. McClung; C. W. Emmons (9976)

N. M. McClung; R. E. Gordon (R-409); J. Caffey

N. M. McClung; R. E. Gordon (R-420 (I)); N. F. Conant (1004)

N. M. McClung; R. E. Gordon (w-3599); S. V. Keating

N. M. McClung; W. Jones $\left(6 \mathrm{M}^{*}\right)$

N. M. McClung; D. Schneidau (306); pulmonary nocardiosis

N. M. McClung; S. McMillan; R. E. Gordon (R-42I);

N. F. Conant (1017)

N. M. McClung; var. crateriforme Bald. CBS

N. M. McClung; C. W. Emmons (NIH) (9935)

N. M. McClung; R. E. Gordon (w-3663); E. Haynes

N. M. McClung; W. Jones $\left(8 \mathrm{~N}^{*}\right)$

N. M. McClung; D. Schneidau (368); S. T. Cowan (I 5)

N. M. McClung; ATCC 4273

* Species name is the name as received.

$\dagger$ Suggested to be a member of $N$. asteroides (see Table Io).

When two groups, Nocardia asteroides (group A) and N. brasiliensis, were compared, the HMO of these showed an S-value of $88 \%$, the highest among the intergroup-Svalues (Table 3). Mean S-value for members of $N$. asteroides to its own HMO was significantly greater than mean S-value to the HMO of another species, $N$. brasiliensis (Table 4). On the other hand, mean S-value for the members of $N$. brasiliensis to its own HMO was significantly larger than that to the HMO of $N$. asteroides (Table 4). These results indicate that $N$. asteroides and $N$. brasiliensis are distinct from each other.

It was noticed that an S-value between two HMOs may differ from an intergroupmean-S-value actually calculated on two groups. As shown in Table 3, S-value between the HMO of Nocardia asteroides (group A) and the HMO of N. brasiliensis was $88 \%$, and S-value between the HMO of $N$. brasiliensis and the HMO of $N$. caviae was $84 \%$. In contrast, when calculated with these groups directly, intergroup-mean S-value between $N$. asteroides (group A) and $N$. brasiliensis was $82 \cdot 9 \pm 3 \cdot 37 \%(n=49)$ 
(Table 5) and intergroup-mean S-value between $N$. brasiliensis and $N$. caviae was $84 \cdot 6 \pm 2 \cdot 62 \%(n=42)$ (Table 6$)$. The former was significantly $(P=5 \%)$ smaller than the latter. It is considered that this discrepancy is due to HMOs being a sym-

\section{Table I2. Strains identified as Nocardia farcinica Trevisan (Trevisan, I889)}

$$
\text { (= group Kyoto- } I=\text { group } B \text { of } N \text {. asteroides) }
$$

\section{Strain*}

N. madurae $\mathrm{C}-407$

$N$. asteroides $\mathrm{M}-72$

$N$. asteroides $\mathrm{M}-\mathrm{I} \mathrm{I} \mathrm{I}$

$N$. eppingeri $\mathrm{M}-112$

$N$. asteroides $\mathrm{M}-\mathrm{I} 3 \mathrm{I}$

$N$. asteroides $\mathrm{M}-\mathrm{I} 37$

$N$. asteroides $\mathrm{M}-138$

$N$. asteroides $\mathrm{M}-139$

$N$. asteroides $\mathrm{M}-\mathrm{I} 44$

$N$. brasiliensis $\mathrm{M}-\mathrm{I} 46$

$N$. asteroides $\mathrm{M}-\mathrm{I} 5^{8}$

$N$. asteroides $\mathrm{M}-86$

$N$. asteroides $\mathrm{M}-\mathrm{I} 27$

$N$. asteroides $\mathrm{M}-\mathrm{I} 33$

$N$. asteroides $\mathrm{M}-\mathrm{I} 58 \mathrm{~A}$

$N$. asteroides $\mathrm{M}-162$

$N$. asteroides $\mathrm{M}-\mathrm{I} 64$

$N$, asteroides $\mathrm{M}-\mathrm{I} 67$

N. brasiliensis M- 169

N. asteroides $\mathrm{M}-\mathrm{I} 77$

$N$. asteroides $\mathrm{M}-\mathrm{I} 79$

$N$. asteroides $\mathrm{M}-\mathrm{I} 8 \mathrm{I}$

$N$. brasiliensis M-197

N. brasiliensis $\mathrm{M}-198$

$N$. brasiliensis $\mathrm{M}-2 \mathrm{OI}$

$N$. asteroides $\mathrm{M}-205$

$N$. asteroides $\mathrm{M}-186$

$N$. asteroides $\mathrm{M}-\mathrm{I} 87$

$N$. asteroides $\mathrm{M}-\mathrm{I} 89$

N. brasiliensis $\mathrm{M}-200$

$N$. madurae C-743

$N$. blackwellii $\mathrm{M}-8 \mathrm{I}$

$N$. asteroides $\mathrm{M}-\mathrm{I} 30$

$N$. asteroides $\mathrm{M}-\mathrm{I} 75$

$N$. asteroides ATCC 3318

$N$. asteroides $\mathrm{W}-3409 \mathrm{~B}$

$N$. asteroides $\mathrm{R}-784$

$N$. asteroides $\mathrm{M}-\mathrm{I} 63 \dagger$

\section{History}

I. Uesaka; Arai; R. E. Gordon (R-407)

N. M. McClung; C. H. Drake; pulmonary nocardiosis

N. M. McClung; R. E. Gordon (R-504)

N. M. McClung; R. E. Gordon (R-508)

N. M. McClung; R. E. Gordon (R-429); N. F. Conant (2 I 23)

N. M. McClung; S. McMillan (N-3I); brain abscess

N. M. McClung; S. McMillan (N-34)

N. M. McClung; S. McMillan (N-35)

N. M. McClung; S. McMillan (0-652)

N. M. McClung; S. McMillan (0-416)

N. M. McClung; Pinkerton, Texas (patient)

N. M. McClung; $N$. farcinica ATCC 33 r 8

N. M. McClung; R. E. Gordon; N. graminis w-360I ; G. J. Friou

N. M. McClung; S. McMillan (N-4)

N. M. McClung; Pinkerton, Texas

N. M. McClung; D. Schneidau (308)

N. M. McClung; D. Schneidau (*3I 2); ATCC 9504

N. M. McClung; D. Schneidau (320)

N. M. McClung; D. Schneidau (327); A. Batista (586)

N. M. McClung; D. Schneidau (343); leg and lung lesions

N. M. McClung; D. Schneidau (345)

N. M. McClung; D. Schneidau (347)

N. M. McClung; D. Schneidau (387)

N. M. McClung; D. Schneidau (390)

N. M. McClung; D. Schneidau (50I); C. Lacaz (299)

N. M. McClung; S. McMillan (N-I2)

N. M. McClung; D. Schneidau (353); A. Batista (702)

N. M. McClung; D. Schneidau (354); Salvin (200I)

N. M. McClung; D. Schneidau (356); (dog)

N. M. McClung; D. Schneidau (397); C. Lacaz (130)

I. Uesaka; Arai; R. E. Gordon (R-743)

N. M. McClung; ATCC 6846

N. M. McClung; R. E. Gordon (R-436); N. F. Conant (2249)

N. M. McClung; D. Schneidau (34I)

R. E. Gordon: originally, $N$. farcinica ATCC 3318

R. E. Gordon; variant of w-3409 (N. polychromogenes;

S. A. Waksman; H. L. Jensen)

R. E. Gordon; B. W. Lacey; A 6 (NCTC)

N. M. McClung; D. Schneidau; Henrici's strain AIOA, var. gypsoides

* Species name is the name as received.

† Suggested to be a member of this species, $N$. farcinica. Remark: the first 30 strains, strain C-407 to strain M-200, are the strains first identified as the group Kyoto-I. Strains, ATCC 33 I 8, W-3409 B and $\mathrm{R}-784$, belonged to the group $\mathrm{B}$ of $N$. asteroides.

bolized feature of a species, prepared by omitting characters appearing at low frequencies. Accordingly, an S-value between two HMOs is not a direct reflexion of an intergroup-mean S-value.

Three examples of comparison between groups are shown in Tables 5, 6 and 7. The 
Table 13. Strains identified as Nocardia brasiliensis (Lindenberg) Pinoy (Pinoy, 1913)

Strain*

N. brasiliensis $\mathrm{R}-405$

$N$. brasiliensis $\mathrm{R}-432$

N. brasiliensis $\mathrm{R}-887$

N. brasiliensis R-III5

$N$. brasiliensis R-I I I7A

N. brasiliensis $\mathrm{R}-\mathrm{I} 188$

$N$. brasiliensis $\mathrm{R}-\mathrm{I} 336$

$N$. brasiliensis $\mathrm{C}-85 \mathrm{I}$

$N$. brasiliensis $\mathrm{M}-\mathrm{I} 99$

N. brasiliensis $\mathrm{M}-204$

N. brasiliensis M-168

N. brasiliensis M-145†
History

R. E. Gordon; Julia M. Coffey; J. B. Fischer; N. F. Conant (N. mexicana)

R. E. Gordon; N. mexicana; I. Christison; N. F. Conant

R. E. Gordon; J. L. Miranda (229)

R. E. Gordon; L. F. Bojalil (N-30); Mycetoma

R. E. Gordon; L. F. Bojalil (variant of N-I I4)

R. E. Gordon

R. E. Gordon

I. Uesaka; Arai; R. E. Gordon (R-85I)

N. M. McClung; D. Schneidau (393); C. Lacaz (602)

N. M. McClung; S. McMillan (N-2)

N. M. McClung; D. Schneidau (322); N. F. Conant (24);

Waksman (3485); G. Ochoa (409)

N. M. McClung; S. McMillan (0-410)

* Species name is the name as received.

$\uparrow$ Suggested to be a member of $N$. brasiliensis (see Table Io).

Table I4. Strains identified as Nocardia caviae (Erikson) Erikson (Erikson, 1935)

Strain*

N. caviae $\mathrm{R}-416$

$N$. caviae $\mathrm{R}-547$

N. caviae R-6I7

N. caviae R-129I

$N$. caviae R-I 315

$N$. caviae R-I3I6

Nocardia $\mathrm{sp} . \mathrm{M}-54$

$N$. asteroides $\mathrm{M}-73$

$N$. asteroides $\mathrm{M}-\mathrm{I} 80$

$N$. asteroides $\mathrm{M}-\mathrm{I} 66$

$N$. asteroides $\mathrm{M}-\mathrm{I} 85$
History

R. E. Gordon; N. asteroides, W. C. Haynes (B-970)

R. E. Gordon; N. asteroides, E. N. Azarowicz

R. E. Gordon; E. N. Azarowicz

R. E. Gordon

R. E. Gordon

R. E. Gordon

N. M. McClung; E. Munch-Peterson

N. M. McClung; NRrL, Peoria

N. M. McClung; D. Schneidau (346); CDC, Atlanta (A-343)

N. M. McClung; D. Schneidau (3I8); CDC, Atlanta (A-343)

N. M. McClung; D. Schneidau (35I); B. H. Webster (I 7292 A)

* Species name is the name as received.

Table I 5. Strains identified as Nocardia pelletieri (Laveran) Pinoy (Pinoy, 1912)

\section{Strain*}

N. pelletieri $\mathrm{R}-408$

N. pelletieri $\mathrm{R}-755$

$N$. pelletieri $\mathrm{R}-755$ var.

N. pelletieri $\mathrm{R}-\mathrm{I} 352$

N. pelletieri $\mathrm{R}-\mathrm{I} 354$

\section{History}

R. E. Gordon; N. pelletieri (Laveran) Pinoy; Julia M. Coffey (47293)

R. E. Gordon; N. pelletieri; J. E. Mackinnon (IHM 614); Lab. de Parasit. Fac. Med. Paris

R. E. Gordon

R. E. Gordon

R. E. Gordon

* Species name is the name as received.

Table I6. Strains identified as Nocardia madurae (Vincent) Blanchard (Blanchard, I896)

Strain*

N. madurae SAL I

N. madurae $\mathrm{R}-43 \mathrm{I}$

N. madurae R-706

$N$. madurae R-I09I

N. madurae R-I 260
History

R. E. Gordon

R. E. Gordon; N. madurae; Isabel Christison; N. F. Conant (2177);

A. González Ochoa (415)

R. E. Gordon; N. madurae; C. W. Emmons (NIH) (9979)

R. E. Gordon; Streptomyces albus; H. D. Tresner (AO-928)

R. E. Gordon; Streptomyces madurae; F. Mariat, Paris (8I2)

* Species name is the name as received. 
Table 17. Frequency of positive characters appearing in each species of Nocardia

No. of strains tested...

I. Gram reaction

2. Acid-fastness, complete

3. Acid-fastness, partial

4. Mycelium, permanent

5. Mycelium, temporary

6. Rod shape (with or without coccoids)

7. Cord formation (compact grouping)

8. Colonial morphology, rough

9. Colonial pigmentation

10. Photochromogenicity

I I. Growth rate, rapid

12. Catalase

13. Nitrate reduction

14. Three-day arylsuiphatase

15. Two-week arylsulphatase

I6. Salicylate degradation

I7. PAS degradation

18. Growth on $0.2 \%$ PAS medium

19. Growth on $\mathrm{NH}_{2} \mathrm{OH}$ medium $(62.5 \mu \mathrm{g} . / \mathrm{ml}$.)

20. Growth on $\mathrm{NH}_{2} \mathrm{OH}$ medium ( $125 \mu \mathrm{g} . / \mathrm{ml}$.)

21. Growth on $\mathrm{NH}_{2} \mathrm{OH}$ medium $(250 \mu \mathrm{g} . / \mathrm{ml}$.)

22. Growth on $\mathrm{NH}_{2} \mathrm{OH}$ medium $(500 \mu \mathrm{g} . / \mathrm{ml}$.)

23. Tolerance to $0.1 \%$ picric acid

24. Tolerance to $0.2 \%$ picric acid

25. Growth at $28^{\circ}$

26. Growth at $37^{\circ}$

27. Growth at $45^{\circ}$

28. Growth at $52^{\circ}$

29. Acetamidase

30. Benzamidase

3I. Urease

32. Isonicotinamidase

33. Nicotinamidaso

34. Pyrazinamidase

35. Salicylamidase

36. Allantoinase

37. Succinamidase

38. Malonamidase

39. Acetate (as $\mathrm{C}$ source)

40. Citrato (as $\mathrm{C}$ source)

41. Succinate (as $C$ source)

42. Malate (as C source)

43. Pyruvate (as $\mathrm{C}$ source)

44. Benzoate (as $C$ source)

45. Malonate (as $\mathrm{C}$ source)

46. Fumarate (as $\mathrm{C}$ source)

47. Acid from glucose

48. Acid from mannose

49. Acid from galactose

50. Acid from arabinose

5I. Acid from xylose

52. Acid from rhamnose
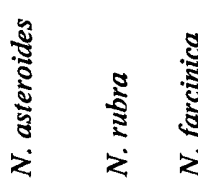

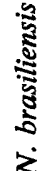

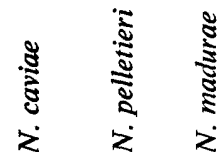

2 I

2 I

0
21

2

2 I

21

2 I

I9

4
0

20

20

21
8

0
8

8

0

2 I

2 I

I7

9

21

20

2 I

I 8

0

2

0

I6

0
I0

$\begin{array}{lll}9 & 38 & 12\end{array}$

$\begin{array}{ll}9 & 38\end{array}$

00

$8 \quad 35$

$0 \quad 9$

$9 \quad 38$

$\begin{array}{ll}9 & 38\end{array}$

9
9

936

$\begin{array}{ll}8 & \text { I } \\ 0 & 0\end{array}$

938

$\begin{array}{ll}9 & 38\end{array}$

00

00

00

00

38

938

935

926

45

$\begin{array}{ll}9 & 38\end{array}$

$9 \cdot 38$

$\begin{array}{ll}9 & 38\end{array}$

$\begin{array}{ll}8 & 38\end{array}$

235

- 6

$\begin{array}{ll}8 & 38\end{array}$

- 27

$5 \quad 37$

00

o 36

o 33

00

0 o

00

$9 \quad 38$

97

935

935

938

$\begin{array}{ll}1 & 0\end{array}$

834

$\begin{array}{ll}8 & 34 \\ 9 & 38\end{array}$

9
5
0

$\begin{array}{ll}0 & 0 \\ 0 & 0\end{array}$

$\begin{array}{rr}0 & 0 \\ 0 & 34\end{array}$

I 2$$
\text { II } 55
$$$$
\text { II } 55
$$$$
\begin{array}{lll}
10 & 5 & 5
\end{array}
$$$$
\text { I } 55
$$$$
\text { II } 55
$$$$
\text { II } 5 \text { 5 }
$$$$
\text { II } 55
$$$$
\text { IO } 1
$$$$
033
$$$$
000
$$$$
\text { II } 000
$$$$
\text { I } 55
$$$$
622
$$$$
000
$$$$
200
$$$$
\text { o } 0 \text { o }
$$$$
0 \text { - - }
$$$$
\text { II - - }
$$$$
\text { II - - }
$$$$
4 \text { - }-
$$$$
\text { I I } 45
$$$$
\text { I I } 35
$$$$
\text { I I } 55
$$$$
\text { I I } 55
$$$$
\text { II } 3
$$$$
000
$$$$
\begin{array}{lll}
0 & 0 & 0
\end{array}
$$$$
\text { II } 0
$$$$
0 \text { o } 0
$$$$
\text { II } 3 \text { I }
$$$$
\text { I I }
$$$$
00
$$$$
7
$$$$
0
$$$$
0
$$$$
\text { I I }
$$$$
\text { o }
$$$$
\text { I I }
$$$$
0
$$$$
\text { I I }
$$

0

0

5

5
4
5

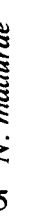


Table I 7 (cont.)

No. of strains tested ...

53. Acid from trehalose

54. Acid from raffinose

55. Acid from inositol

56. Acid from mannitol

57. Acid from sorbitol

58. Glycerol (as C source)

59. Glucose (as C source)

60. Mannose (as C source)

61. Galactose (as C source)

62. Arabinose (as $\mathrm{C}$ source)

63. Xylose (as C source)

64. Rhamnose (as C source)

65. Trehalose (as C source)

66. Raffinose (as C source)

67. Inositol (as C source)

68. Mannitol (as C source)

69. Sorbitol (as C source)

70. Fructose (as C source)

71. Sucrose (as C source)

72. Ethanol (as C source)

73. Propanol (as $\mathrm{C}$ source)

74. Propylene glycol (as C source)

75. I,3-Butylene glycol (as $\mathrm{C}$ source)

76. I,4-Butylene glycol (as C source)

77. 2,3-Butylene glycol (as $\mathrm{C}$ source)

78. L-Glutamate (as $\mathrm{N}-\mathrm{C}$ sources)

79. L-Serine (as N-C sources)

8o. Glucosamine (as N-C sources)

81. Acetamide (as N-C sources)

82. Benzamide (as N-C sources)

83. Monoethanolamine (as N-C sources)

84. Trimethylene diamine (as $\mathrm{N}-\mathrm{C}$ sources)

85. L-Glutamate (as $\mathrm{N}$ source)

86. L-Serine (as N source)

87. L-Methionine (as $\mathrm{N}$ source)

88. Acetamide (as N source)

89. Benzamide (as N source)

9o. Urea (as $\mathrm{N}$ source)

91. Pyrazinamide (as N source)

92. Isonicotinamide (as $\mathrm{N}$ source)

93. Nicotinamide (as $\mathrm{N}$ source)

94. Succinamide (as N source)

95. Nitrate (as N source)

96. Nitrite (as $\mathrm{N}$ source)

97. Nicotinic acid

98. Growth on TCH medium (Io $\mu \mathrm{g} . / \mathrm{ml}$.)

99. Growth on salicylate medium ( $0.05 \%)$

100. Growth on salicylate medium ( $(0.1 \%)$

Mean no. of positive characters per strain

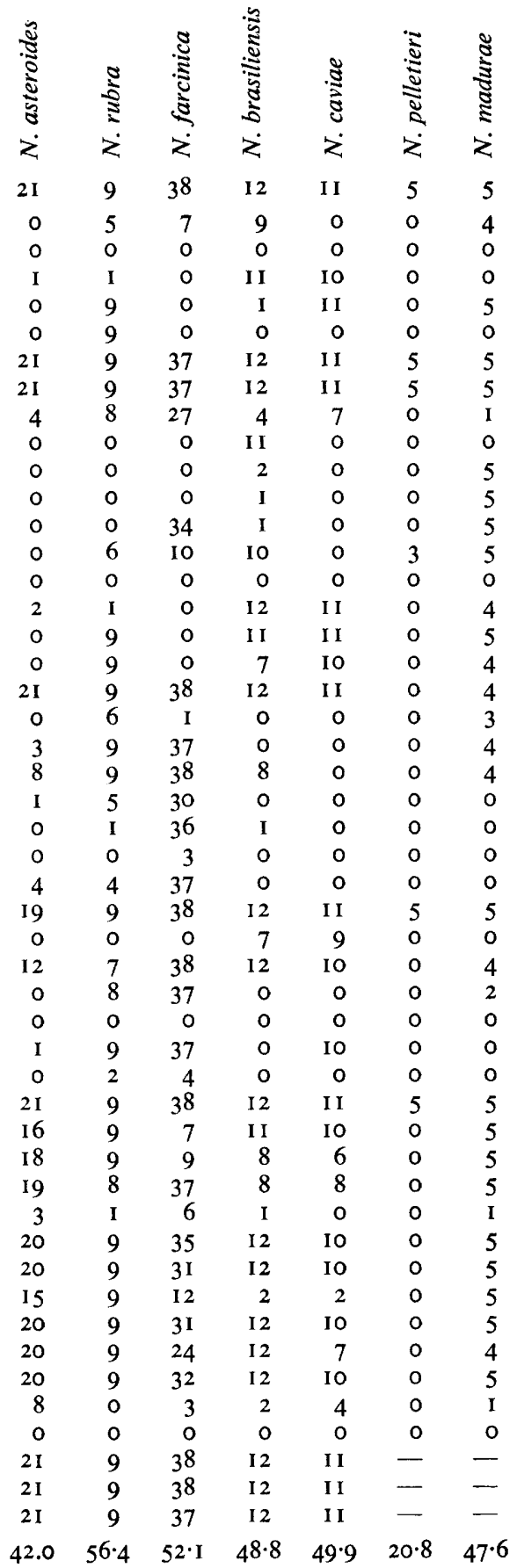


comparisons, made by the method described by Tsukamura ( $1967 b$ ) showed that the groups tested were two different clusters.

To return to the problem of Nocardia farcinica, that is, group B of $N$. asteroides. Group Kyoto-I, consisting of 30 strains received from Dr Uesaka, formed one large

\section{Table 18. Strains not identified as any of 7 species}

Strain

N. coeliaca $\mathrm{M}-\mathrm{I} 22$

$N$. transvalensis M-193

N. tenuis convoluta $\mathrm{M}-203$

N. restrictus $\mathrm{M}-9$

N. bostroemi $\mathrm{M}-\mathrm{I} 59$

N. intracellularis $\mathrm{M}-\mathrm{I} 70$

N. madurae $\mathrm{M}-\mathrm{I} 72$

N. madurae $\mathrm{C}-637$

N. leishmani $\mathrm{M}-82$

N. alba (schoen) M-I 13

$N$. asteroides $\mathrm{M}-123$
History

N. M. McClung; C. W. Emmons (NIH)

N. M. McClung; D. Schneidau (366); R. E. Gordon (366); N.C.T.C.

N. M. McClung; D. Schneidau (55I); A. Batista (I89)

N. M. McClung; CBS; (= R-566)

N. M. McClung; D. Schneidau (303); A. Batista (630); Inst.

Pasteur, Paris (32I); (= R-IOI I)

N. M. McClung; D. Schneidau (328); M. L. Littman; Wiley

N. M. McClung; D. Schneidau (333); N. F. Conant (21 77); G. Ochoa

I. Uesaka; Arai; R. E. Gordon (R-637); A. G. Ochoa (486)

N. M. McClung; ATCC 6855; lung disease and pericarditis

N. M. McClung; R. E. Gordon (R-506); Inst. Pasteur, Paris

N. M. McClung; R. E. Gordon (w-366I); C. A. Payne; brain abscess

Table I9. Typical strains for the species of Nocardia

\begin{tabular}{|c|c|c|}
\hline Species & $\begin{array}{l}\text { Typical strain } \\
\text { (species name as } \\
\text { received is shown) }\end{array}$ & $\begin{array}{l}\text { S-value to } \\
\text { e HMO of } \\
\text { ts species } \\
\quad(\%)\end{array}$ \\
\hline $\begin{array}{l}N . \text { farcinica (group } \\
\text { Kyoto-I; group B } \\
\text { of ' } N . \text { asteroides') }\end{array}$ & $\begin{array}{l}\text { N. asteroides M-I33 } \\
\text { (ATCC 23825) } \\
\text { N. asteroides M-205 } \\
\text { (ATCC 23826) } \\
\text { N. asteroides M-175 }\end{array}$ & $\begin{array}{l}99 \\
99 \\
97\end{array}$ \\
\hline $\begin{array}{l}\text { N. rubra (group } \\
\text { Kyoto-II) }\end{array}$ & $\begin{array}{l}\text { N. rubra } \mathrm{M}-\mathrm{I} \\
N . \text { globerula } \mathrm{M}-75 \\
\text { N. lutea } \mathrm{M}-\mathrm{I} 92\end{array}$ & $\begin{array}{l}97 \\
97 \\
97\end{array}$ \\
\hline $\begin{array}{l}\text { N. asteroides (group A } \\
\text { of } N \text {. asteroides) }\end{array}$ & $\begin{array}{l}N . \text { asteroides } \mathrm{R}-399^{*}(\mathrm{ATCC} 23824) \\
N . \text { asteroides } \mathrm{M}-\mathrm{I} 29 \\
N . \text { asteroides ATCC } 9970 \\
\text { (Gordon) }\end{array}$ & $\begin{array}{l}99 \\
97 \\
95\end{array}$ \\
\hline N. brasiliensis & $\begin{array}{l}N . \text { brasiliensis } \mathrm{R}-432 \\
N . \text { brasiliensis } \mathrm{R}-887\end{array}$ & $\begin{array}{r}100 \\
98\end{array}$ \\
\hline$N$. caviae & $\begin{array}{l}N . \text { caviae } \mathrm{R}-\mathrm{I} 3 \mathrm{I} 5 \\
N . \text { caviae } \mathrm{R}-\mathrm{I} 3 \mathrm{I} 6 \\
N . \text { caviae } \mathrm{R}-4 \mathrm{I} 6\end{array}$ & $\begin{array}{l}99 \\
99 \\
98\end{array}$ \\
\hline N. pelletieri & $\begin{array}{l}N . \text { pelletieri } \mathrm{R}-\mathrm{I} 352 \\
N . \text { pelletieri } \mathrm{R}-408\end{array}$ & $\begin{array}{l}98 \\
98\end{array}$ \\
\hline N. madurae & $\begin{array}{l}\text { N. madurae SAL I } \\
\text { N. madurae R-706 }\end{array}$ & $\begin{array}{l}95 \\
94\end{array}$ \\
\hline
\end{tabular}

* Proposed neotype strain deposited in the American Type Culture Collection, Rockville, Maryland, 20852, U.S.A.

cluster in numerical classification; characters of this group seemed to be similar to those of the group B of $N$. asteroides ( $N$. farcinica). Twenty-two of the 30 strains had been received as $N$. asteroides, and the group Kyoto-I included strain M-86 of $N$. asteroides, which is the same as strain ATCC 3318 of $N$. farcinica. 
Identification of the group B of Nocardia asteroides (that is, N. farcinica) as group Kyoto-I was carried out by determining S-values for three strains of the group B to various HMOs. These are shown in Table 8. Assuming that S-values to the HMO for individual members of a species show a normal distribution curve, it will be expected that $c .95 \%$ of the members of a species will be included within the limits ((Mean) \pm $2 \times$ (Standard deviation)). Thus, a permissible low limit of the S-value for strains to be included in a species will be ((Mean) $-2 \times($ Standard deviation $)) \%$; this limit is calculated from the data of Table 2 and is shown in Table 8.

Table 20. Pattern of utilization for growth of nitrogen compounds as simultaneous nitrogen and carbon sources and pattern of amidases in the species of Nocardia

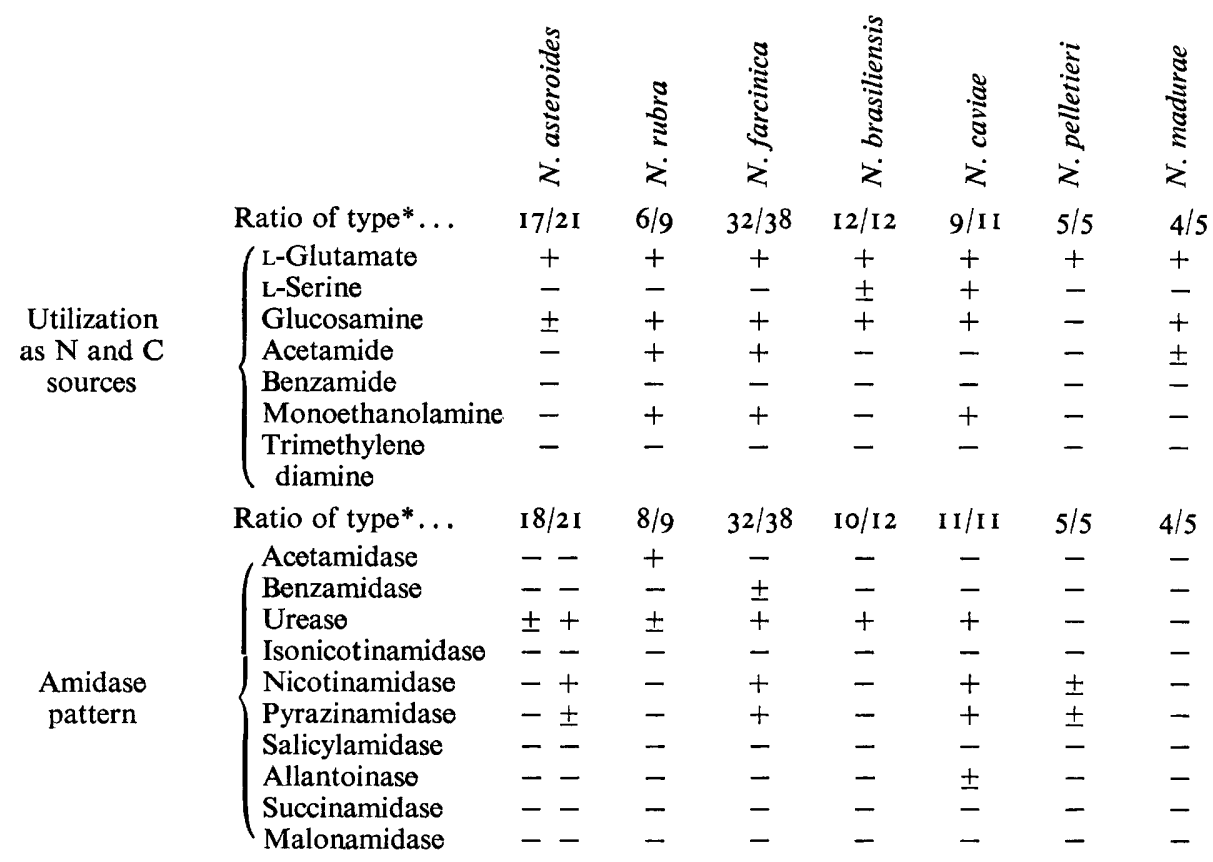

* (No. of strains showing the pattern indicated)/(no. of strains tested).

When S-values for the 3 strains of group B to various HMOs were determined, only S-values to the HMO of group Kyoto-I were within the permissible low limit $(88.3 \%$ or more); S-values to the other HMOs remained outside the limits (Table 8). Thus, strains ATCC 33I8, W-3409 B and R-784 were identified as a member of the group Kyoto-I.

Nomenclature of species. From the results obtained, 7 groups could be recognized in this study: (I) Kyoto-I = group B of Nocardia asteroides; (2) Kyoto-II; (3) group A of $N$. asteroides; (4) N. brasiliensis; (5) N. caviae; (6) N. pelletieri; (7) N. madurae. Of these, the nomenclature of the last four is no problem. In the following, nomenclature of the first three is discussed.

Strains of group A of Nocardia asteroides and group B of $N$. asteroides (= Kyoto-I) had been received bearing the name $N$. asteroides. These two groups, however, showed distinctly different clusters and were considered to be two different species. According 
to the description of Gordon \& Mihm (I957, I959, I962a), the group A seemed to be correctly named $N$. asteroides (Eppinger) Blanchard. This was discussed previously.

Group B (Kyoto-I) has been named Nocardia farcinica Trevisan for the following reasons: (a) Gordon \& Mihm (1957, 1962a) recognized the priority of the name $N$. farcinica over the name $N$. asteroides, but they avoided the name $N$. farcinica because they considered that the name $N$. asteroides had already become established by usage; $(b)$ the group containing strain ATCC 33 I 8 was recognized as a distinct cluster clearly differentiated from other groups of Nocardia, including N. asteroides group A.

Table 21. Distinguishing characters for the species of Nocardia

Character

Growth at $45^{\circ}$

Acetamidase

Benzamidase

Urease

Malate as $\mathrm{C}$ source

Succinate as $\mathrm{C}$ source

$\mathrm{I}, 3$-Butylene glycol as $\mathrm{C}$ source

Acid from arabinose

Acid from galactose

Acid from inositol

Acid from mannitol

Acid from rhamnose

Acid from sorbitol

Serine as $\mathrm{N}$ and $\mathrm{C}$ sources

Glucosamine as $\mathrm{N}$ and $\mathrm{C}$ sources

Acetamide as $\mathrm{N}$ and $\mathrm{C}$ sources

Monoethanolamine as $\mathrm{N}$ and $\mathrm{C}$ sources

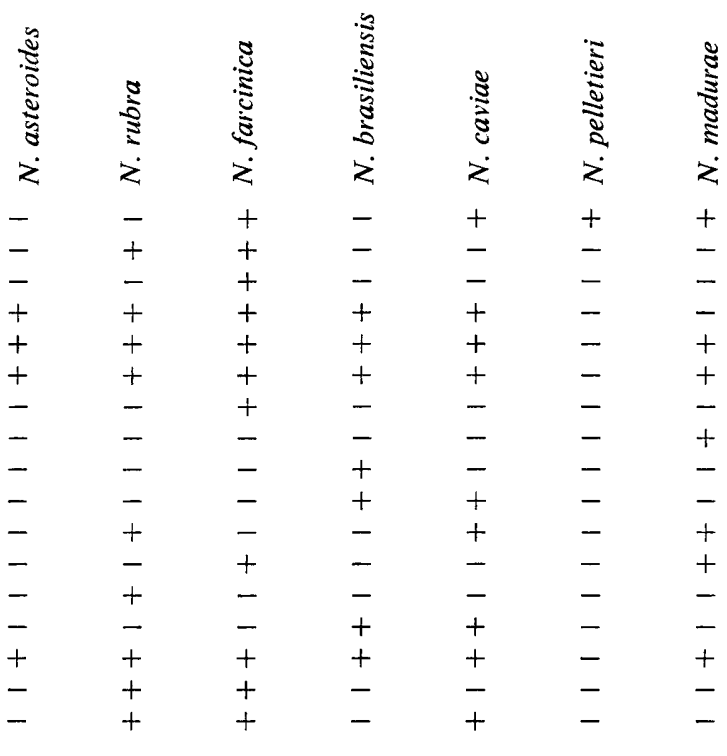

The group Kyoto-II included 7 strains. Two strains were identified later, and a total of 9 strains of this group is listed in Table 9. Among these, the following 5 had been identified by Gordon \& Mihm (1957) as Mycobacterium rhodochrous: (I) N. rubra M-I; (2) N. erythropolis $\mathrm{M}-8$; (3) N. globerula $\mathrm{M}-75$; (4) N. lutea $\mathrm{M}-192$; (5) N. polychromogenes M-6. One strain, $N$. minima M-I03, had been identified by them as $N$. asteroides. Accordingly, this group might be identified as $M$. rhodochrous, but it showed several discrepancies from the characters of that species as described by Gordon \& Mihm (I957). It is possible that, since the strains used by us had been transferred through two or three investigators and had been maintained in $\mathrm{Dr}$ Uesaka's laboratory for 7 years, some strains might be mislabelled or have changed their characters. Considering this possibility, strain ATCC I3808 of $M$. rhodochrous received directly from the American Type Culture Collection was studied and compared with the above strains. ATCC I 3808 showed an S-value of $86 \%$ to the HMO of the group Kyoto-II, a value outside the permissible limit for the group. This result made us hesitate to identify this group as $M$. rhodochrous, and we preferred to name it, provisionally, Nocardia rubra. This name had appeared first among 8 names for 
this group, $N$. rubra, $N$. erythropolis, $N$. globerula, $N$. convoluta, $N$. minima, $N$. lutea, N. polychromogenes and N. rubropertincta (Bergey's Manual, 1957; Index Bergeyana, 1966).

\section{Identification of strains not forming a cluster by preliminary sorting}

It is supposed that, when S-values to the HMO for individual members of a species show a normal distribution curve, $c .95 \%$ of the members of the species are included to the range $\{$ (mean) $\pm 2 \times$ (standard deviation) $\}$. The permissible low limit of the $\mathrm{S}$-value to an HMO is set for each species at (mean S-value to the HMO) $-2 \times$ (standard deviation) $\} \%$. After establishing the permissible low limit for species, identification is made without subjective judgement (automatically) by determining $\mathrm{S}$-values for the unknown strain to various HMOs. If an S-value to a HMO is within the permissible limit and S-values to the other HMOs are outside the permissible limits, the unknown strain is identified as a species of the first HMO. This method was applied to the strains received from Dr Uesaka and not forming a cluster in preliminary sortings, and the results are shown in Table Io. Adding the strains thus identified, the strains identified in the 7 species are shown in Table 9 and Tables II to I6, and the frequency of characters in the species summarized in Table $\mathbf{I} 7$.

Eleven strains remained unidentified (Table 18 ); some may be distinct species. S-values between these strains were less than $80 \%$, except in the following pairs: (I) Nocardia intracellularis $\mathrm{M}-\mathrm{I} 70$ and $N$. bostroemi M-I $59(88 \%)$; (2) N. intracellularis M-I70 and $N$. restricus M-9 $(84 \%)$; (3) N. leishmanii M-82 and N. alba M-I I3 (87\%); (4) N. madurae C-637 and N. coeliaca M-I $22(83 \%)$.

Typical strains of the species. Strains showing a high similarity to the HMO are used as reference strains, and are shown in Table 19.

\section{A simple method of identification}

The principles of numerical taxonomy make an ideal basis for a method of identification, and a method of using HMOs has been presented in this paper. Laboratories not specialized for bacterial identification need a simpler method. In the identification of mycobacteria, two methods have been found useful, amidase pattern (Bönicke, 1962) and pattern of nitrogen compounds used as simultaneous nitrogen and carbon sources (Tsukamura, I $967 c$ ). By a combination of these two methods, identification is successful with most strains, and typical patterns of the species are shown in Table 20.

Schneidau (1963) stated that the amidase pattern was not as uniform in nocardias as in mycobacteria, and in the present study it was found that identification of nocardias was not successful by the use of the amidase pattern alone, but the test was useful in combination with utilization of nitrogen compounds as simultaneous nitrogen and carbon sources. A practical diagnostic table was prepared by a selection of distinguishing characters, with frequencies of more than $9 \mathrm{I} \%$ in some species but in others at a frequency as low as $10 \%$. This is shown in Table $2 \mathrm{I}$, which included a few characters with a frequency less than $90 \%$.

Although both identification tables (Tables 20 and 2I) include 17 tests, Table 20 could be more easily applied in practice. Table $2 \mathrm{I}$ contains more characteristic tests, and would provide a more accurate result. 


\section{DISCUSSION}

Division of Nocardia asteroides into two species

The most important conclusion reached in this study is that the species $N$. asteroides can be divided into two species, Nocardia asteroides (Eppinger) Blanchard and Nocardia farcinica Trevisan (both in new senses). Among I 2 strains received as Nocardia species, 2I strains of $N$. asteroides (in a new sense) and 38 strains of $N$. farcinica (in a new sense) were identified (Tables II and I2); the data of comparison between these two species are summarized in Table 22. Eighteen of 2 I strains of the former and 28 of 38 strains of the latter had been named as $N$. asteroides when received.

Table 22. Comparison between Nocardia asteroides (group A of N. asteroides) and N. farcinica (group B of N. asteroides; group Kyoto-I)

\begin{tabular}{|c|c|c|c|}
\hline \multirow[b]{2}{*}{ Character } & \multicolumn{2}{|c|}{$\begin{array}{l}\text { No. of strains showing } \\
\text { a positive reaction* }(\%)\end{array}$} & \multirow{2}{*}{$\begin{array}{l}N . \text { asteroides } \\
\text { according to the } \\
\text { description of } \\
\text { Gordon \& Mihm } \\
\text { (1962) }\end{array}$} \\
\hline & N. asteroides & N. farcinica & \\
\hline No. of strains tested... & $2 \mathrm{I}$ & 38 & 142 \\
\hline+ Growth at $45^{\circ}$ & $o(0)$ & $35(92)$ & $(42)$ \\
\hline$†$ Acetamidase & $2(\mathrm{IO})$ & $38(100)$ & . \\
\hline$\dagger$ Benzamidase & $0(0)$ & $27(7 \mathrm{I})$ & . \\
\hline Pyrazinamidase & $4(19)$ & $33(87)$ & . \\
\hline$\dagger$ Acid from rhamnose & $o(0)$ & $34(90)$ & (32) \\
\hline Rhamnose as $\mathrm{C}$ source & $o(0)$ & $34(90)$ & . \\
\hline Ethanol as $\mathrm{C}$ source & $3(14)$ & $37(87)$ & . \\
\hline Propylene glycol as $\mathrm{C}$ source & I $(5)$ & $30(79)$ & . \\
\hline$\uparrow \mathrm{I}, 3$-Butylene glycol as $\mathrm{C}$ source & $o(0)$ & $36(95)$ & . \\
\hline 2,3-Butylene glycol as C source & 4 (I9) & $37(97)$ & . \\
\hline$\dagger$ Acetamide as $\mathrm{N}$ and $\mathrm{C}$ sources & $o(0)$ & $37(97)$ & . \\
\hline$\dagger$ Monoethanolamine as $\mathrm{N}$ and $\mathrm{C}$ source & ce $1(5)$ & $37(97)$ & . \\
\hline Nitrate reduction & $8(38)$ & $0(0)$ & $(88)$ \\
\hline Two week-arylsulphatase & $8(38)$ & $o(0)$ & . \\
\hline
\end{tabular}

Relationships between species. Numerical classification of the strains received from Dr Gordon differentiated 6 clusters, (I) Nocardia asteroides, (2) N. farcinica, (3) $N$. brasiliensis, (4) N. caviae, (5) N. pelletieri, and (6) N. madurae (Fig. I), and these results agreed well with the results obtained by classical methods, except that $N$. asteroides was divided into two species, $N$. asteroides (in a new sense) and $N$. farcinica. Numerical classification of the strains received from Dr Uesaka differentiated another cluster of $N$. rubra. Relationships between these species are shown in Table 3 as an S-value table of HMOs of these species and in Fig. 4 as a dendrogram.

The roo characters used in this study were mainly composed of nutritional requirement tests and biochemical tests, and included only 9 morphological characters. Since morphological characteristics have been recognized as important in classical taxonomy of the genus Nocardia, we would like to add some comments on morphology. All strains received from Dr Gordon as $N$. madurae and $N$. pelletieri showed well-developed mycelium tending to fragmentation (designated permanent mycelium in this study). They showed only scant growth on Ogawa egg medium, possibly due to their sus- 
ceptibility to malachite green (Uesaka, 1964), making it impossible to take into account in the S-value characters determined on this medium. These two species were distinct from other species in dendrograms (Fig. 2 and 4) and seemed to belong to a Streptomyces-like nocardia of Schneidau \& Shaffer (1957). However, it has not been concluded whether or not these are Streptomyces.

Permanent mycelium was observed not only in these species but also, to some extent, in I6 strains of other species. Among these, 5 belonged to an unidentified group (Table I8) received as $N$. madurae $\mathrm{C}-637, N$. leishmanii $\mathrm{M}-82, N$. alba $\mathrm{M}-\mathrm{I}$ I3, $N$. asteroides $\mathrm{M}-\mathrm{I} 23$ and $N$. madurae $\mathrm{M}-\mathrm{I} 72$. One belonged to $N$. caviae (M-I80 received as $N$. asteroides), 2 belonged to $N$. asteroides (M-94 and $\mathrm{M}-\mathrm{I} 6 \mathrm{I}$ received as $N$. asteroides), and 8 belonged to $N$. farcinica (M-72, M-I $58 \mathrm{~A}, \mathrm{M}-\mathrm{I} 62$, M-I67, M-I69, M-I 77, M-I79 and M-I8I received as $N$. asteroides). The presence of permanent mycelium seemed not to be sufficient to differentiate $N$. pelletieri and $N$. madurae from other species.

When observed by naked eye on glucose agar after incubation at $28^{\circ}$ for 4 weeks aerial hyphae were observed at the following rates: $N$. asteroides, 5/2I $(24 \%)$; N. farcinica, 10/38 (26\%); N. brasiliensis, $2 / 12($ I $7 \%) ; N$. caviae, I/I I (9\%); N. rubra, o/9 (0\%); N. pelletieri, o/5 (0\%); N. madurae, o/5 (0\%); unidentified, $2 / \mathrm{I}$.

The author thanks greatly Dr Ruth E. Gordon, Institute of Microbiology, Rutgers, The State University, New Brunswick, New Jersey, U.S.A., and Dr I. Uesaka, Tuberculosis Research Institute, Kyoto University, Kyoto, Japan, for their kind supply of test strains and for their encouragement. He thanks also Dr Erwin F. Lessel, the American Type Culture Collection, Rockville, Maryland, U.S.A., for his advice. He acknowledges with thanks technical assistance of S. Mizuno, H. Toyama, K. Okada and M. Oshima.

\section{REFERENCES}

Bergey's Manual of Determinative Bacteriology (1957). 7th ed. Ed. by R. S. Breed, E. G. D. Murray and N. R. Smith, p. 7I5. Baltimore: The Williams and Wilkins Co.

Blanchard, R. (I896). Parasites végétaux a l'exclusion des bactéries. In Blanchard, C. (I896). Traité de Pathologie Générale. G. Masson: Paris. 2, 81 I (cited from Index Bergeyana).

BöNICKE, R. (1962). L'identification des mycobactéries a l'aide des méthodes biochimiques. Bull. Un. int. Tuberc. 32, 13.

Erikson, D. (1935). The pathogenic aerobic organisms of the Actinomyces group. Spec. Rep. Ser. med. Res. Coun. 203, 5.

Gordon, R. E. (I966a). Some criteria for the recognition of Nocardia madurae (Vincent) Blanchard. J. gen. Microbiol. 45, 355 .

Gordon, R. E. (1966b). Some strains in search of a genus-Corynebacterium, Mycobacterium, Nocardia or what? J. gen. Microbiol. 43, 329.

Gordon, R. E. \& Mirm, J. M. (1957). A comparative study of some strains received as nocardiae. J. Bact. 73, I5.

Gordon, R. E. \& Miнm, J. M. (1959). A comparison of Nocardia asteroides and Nocardia brasiliensis. J. gen. Microbiol. 20, 129.

Gordon, R. E. \& Miнm, J. M. (1962a). The type species of the genus Nocardia. J. gen. Microbiol. 27, I.

Gordon, R. E. \& Mirm, J. M. (1962b). Identification of Nocardia caviae (Erikson) nov. comb. Ann. N.Y. Acad. Sci. 98, 628.

Liston, J., Wiebe, W. \& Colwell, R. R. (1963). Quantitative approach to the study of bacterial species. J. Bact. 85 , 106I.

Pinoy, E. (1913). Actinomycoses et mycétomes. Bull. Inst. Pasteur I1, 929. 
PINOY, M. (I9I2). Mycétome à grains rouges de la paroi thoracique. Isolement et culture d'une nouvelle Oospora pathogène. Bull. Soc. Path. Exot. 5, 585 (cited from Index Bergeyana).

SCHNEIDAU, J. D. Jun. (1963). The amidase activity of certain species of Nocardia and Mycobacterium. Am. Rev. resp. Dis. 88, 563.

Schneidau, J. D. Jun. \& Shaffer, M. F. (1957). Studies on Nocardia and other Actinomycetales. I. Cultural studies. Am. Rev. Tuberc. pulm. Dis. 76, 770.

SNEATH, P. H. A. (1957a). Some thoughts on bacterial classification. J. gen. Microbiol. 17, I84.

SNeAth, P. H. A. (1957 b). The application of computers to taxonomy. J. gen. Microbiol. 17, 20 I.

SNeAth, P. H. A. (1964). New approaches to bacterial taxonomy: Use of computers. A. Rev. Microbiol. $18,335$.

SNEATH, P. H. A. \& Cowan, S. T. (1958). An electro-taxonomic survey of bacteria. J. gen. Microbiol. 19, 55 I.

Sokal, R. R. \& SNeath, P. H. A. (1963). Principles of Numerical Taxonomy. San Francisco: W. H. Freeman Co.

Trevisan, J. (1899). I generi e le specie delle batteriacee. Milano pp. I-35 (cited from Index Bergeyana).

TsuKamura, M. (1966). Adansonian classification of mycobacteria. J. gen. Microbiol. 45, 253.

TsuKamura, M. (1967a). Identification of mycobacteria. Tubercle, Lond. 48, $3 \mathrm{II}$.

Tsukamura, M. (1967b). A statistical approach to the definition of bacterial species. Jap. J. Microbiol. II, 213.

TsuKamuRA, M. (1967c). Differentiation of mycobacteria by utilization of nitrogen compounds as simultaneous nitrogen and carbon sources. Am. Rev. resp. Dis. 95, 307.

Tsukamura, M. \& Mizuno, S. (1968). 'Hypothetical Mean Organisms' of mycobacteria. A study of classification of mycobacteria. Jap. J. Microbiol. 12, $37 \mathrm{I}$.

UeSAKA, I. (1964). The tolerance of actinomycetes to acid, alkali, and malachite green as an aid to their classification and its application to the isolation of Nocardia from sputa. Acta tuberc. Jap. r4, I.

NOTE

After preparation of the manuscript (May 1968), the author was informed that there was a paper of Cerbón (1967) dealing with numerical taxonomy of Nocardia.

Recently, Magnusson \& Mariat (I968) stated that $N$. asteroides seemed to be a separable species by an immunological method based on the specificity of delayed type skin reactions on guinea pigs. Their results are compatible with our results.

\section{REFERENCES}

Cerbón, J. (1967). Taxonomic analysis of Nocardia. Revta. lat.-am. Microbiol. Parasit. 9, 65. MAGNuSsON, M. \& MARIAT, F. (I968). Delineation of Nocardia farcinica by delayed type skin reactions on guinea pigs. J. gen. Microbiol. 5r, I5I. 